\title{
Separating Distribution from Coordination and Computation as Architectural Dimensions
}

\author{
José Luiz Fiadeiro \\ Department of Computer Science, University of Leicester \\ University Road, Leicester LE1 7RH, UK \\ jose@mcs.le.ac.uk
}

The power of architectural modelling approaches in addressing the complexity of software systems derives, to a large extent, from the way they are able to separate coordination from computation concerns. However, distribution has become a key factor of complexity in the modelling of ubiquitous, software-intensive systems. Distribution interferes with both the way computations are performed and interactions are coordinated. Can we separate it as a third architectural dimension? If so, how can we derive the joint behaviour that emerges when the three dimensions are brought together?

In this talk, we provide an overview of our joint work with Dr. Antónia Lopes, from the University of Lisbon, around CommUnity - a prototype language for architectural description that provides a formal framework in which the questions above can be formulated and answered in general mathematical terms. 